\title{
Magnetic sensor data acquisition for three- dimensional ultrasound of the orbit
}

\section{Abstract}

Purpose Three-dimensional (3D) B-mode ultrasound imaging is now possible using a magnetic sensor data acquisition system to localise data points, allowing free-hand scanning. We report the first use of this freehand scanning system for insonation of the orbit.

Methods We insonated 10 orbits in 5 volunteers (all men; mean age 37 years) with an Acuson 128XP ultrasound system and a $7.5 \mathrm{MHz}$ transducer. Power-Doppler was used to image vascular structures. Data were acquired using a new magnetic sensor system with detectors mounted on the transducer. Free-hand scanning was done using sweeping or fan-like and linear movements over 20-30 s to cover the orbit, with retrospective measurements of orbital structures. A 3D reconstruction was performed via an extemal workstation. Results All orbits were adequately imaged by one data acquisition. Selected intraorbital structures were identified and their course followed, including optic nerve and central vessels, as well as short and long ciliary vessels. The mean transverse area of the optic nerve was $5.6 \pm 1.1 \mathrm{~mm}^{2}$; the diameter of the optic nerve was $3.0 \pm 0.3 \mathrm{~mm}$. The lateral rectus muscle was clearly seen, with a mean diameter of $4.9 \pm 0.3 \mathrm{~mm}$, at the level of optic nerve head. Conclusion Three-dimensional ultrasound of the orbit allows imaging of the location, course and relationships of intraorbital vessels, the optic nerve and the lateral rectus muscles with one data acquisition, as well as quantitative measurements not possible with 2D images alone. This method allows rapid, free-hand data acquisition, with a multitude of potential clinical applications.

Key words Colour Doppler ultrasonography, Free-hand scanning, Optic nerve, Orbit, Threedimensional ultrasound

Conventional two-dimensional (2D) ultrasound is used for visualising the topography of ocular and orbital tissue. Mainly, two different ranges of ultrasound frequencies have been used: higher frequencies of up to $50 \mathrm{MHz}$ are used to examine the anterior segment, while lower frequencies of $7.5-10.0 \mathrm{MHz}$ are used to perform ophthalmic ultrasound of single structures of the total orbit. ${ }^{1}$ B-scan of the orbit with lower frequencies can show the relationship of the optic nerve to normal structures such as the extraocular muscles and orbital bones, as well as to retrobulbar lesions. Colour Doppler ultrasound imaging is a noninvasive ultrasound procedure that permits simultaneous grey-scale imaging of structure and colour-coded imaging of blood velocities, used to examine intraorbital vessels. ${ }^{2-4}$ Twodimensional B-mode imaging gives incomplete information about the orbital anatomy and pathology since it can only evaluate a single anatomical plane. Three-dimensional

reconstruction of $2 \mathrm{D}$ ultrasound data is useful to fully represent the anatomical relationships in the orbit. A great advantage of 3D ultrasound is documentation of all 2D ophthalmic ultrasound sections in a 3D data set. This avoids section artefacts, which can limit 2D studies, and offers the option of a retrospective review of any scan plan to provide optimal visualisation of structures. This improves the consistency of readings, even among different sonographers or readers. Another advantage is the documentation of ultrasound images for the comparison with follow-up examinations and volume measurements (e.g. tumours of the orbit). Three-dimensional ultrasound imaging of the orbit has been previously reported, but with a mechanical data acquisition system (see Table 2) ${ }^{5-10}$ With mechanical data acquisition, 3D scan sets are obtained by acquiring multiple 2D B-scans separated by fixed linear intervals or rotational angles. ${ }^{8,11}$ However, mechanical systems limit the mobility of the transducer during the examination. The advantage of magnetic sensor data acquisition is that the 3D ultrasound data can be obtained by free-hand scanning.

\section{Patients and methods}

We insonated 10 orbits of five volunteers (all men; mean age 37 years) with a magnetic sensor
A. Delcker

Department of Neurology Neurosonology Laboratory Bowman Gray School of Medicine

Wake Forest University

Winston-Salem North Carolina, USA

T. Martin

Department of

Ophthalmology

Bowman Gray School of Medicine

Wake Forest University Winston-Salem

North Carolina, USA

Andreas Delcker, M.D. Department of Neurology University of Essen Hufelandstrasse 55 D-45122 Essen Germany

Tel: +49 2017232588 Fax: +49 2017233797 
3D data acquisition system (3D EchoTech, Munich, Germany). All volunteers gave their informed consent. The scans were performed with the subject lying supine, eyelids closed, directing their gaze towards the ceiling.

\section{Data acquisition}

Data acquisition through the orbit was performed using the magnetic sensor system to track the spatial orientation of the ultrasound probe while scanning the volume of interest. ${ }^{12} \mathrm{~A}$ magnetic field is generated from an array of three electrical coils that are at $90^{\circ}$ to each other, each aligned differently and phase shifted but with the same frequency. The combined effect of the three magnetic fields is an inhomogeneous magnetic field that has a 3D orientation. A magnetic sensor array, consisting of three small magnetic coils, lying in different planes, is attached to the transducer. This allows the system to detect the positioning of the transducer relative to the source of the magnetic field with accurate spatial localisation of the 2D ultrasound data in a 3D data set. Imaging was achieved through the orbit with fan-like and transverse movements of the transducer through the orbit, obtaining transverse orbit sections. The data acquisition was performed to allow optimal insonation of the optic nerve in the centre of the field of view of the 2D image. With this movement of the transducer the lateral rectus muscle was integrated in the ultrasound sections. The total duration of data acquisition for a complete 3D reconstruction was 20-30 s. After data acquisition we stored a complete $3 \mathrm{D}$ data set of the ultrasound sections of the orbit. One data acquisition was performed with grey-scale B-mode and a second with power mode
Doppler was performed for the evaluation of the intraorbital vessels.

\section{Ultrasound system}

For imaging of the orbit an Acuson 128XP duplex system with a $7.5 \mathrm{MHz}$ sector transducer (Acuson, Mountain View, CA) was used. Power Doppler was used to image vascular structures. Insonation depth was limited to $60 \mathrm{~mm}$ to maintain acceptable B-mode resolution of the anterior and middle orbit. Copious amounts of ultrasound coupling gel facilitated good visualisation of structures in the orbit. The 2D B-mode ultrasound image was optimised by altering the time-gain curve, field of view and overall gain settings.

\section{Three-dimensional reconstructions}

The magnetic sensor system allowed the 3D workstation to create $3 \mathrm{D}$ reconstructions (Fig. 1) and 3D images of the orbit. The $3 \mathrm{D}$ reconstruction time was $1-2 \mathrm{~min}$.

\section{Measurement method}

The diameter and area of the optic nerve were measured in a transverse section immediately behind the globe. For the diameter measurement the maximal diameter of the optic nerve, and for area measurements the outlines of the optic nerve, were marked, and the area was calculated in square millimetres. The diameter of the lateral rectus muscle was measured at the level of the optic nerve head.

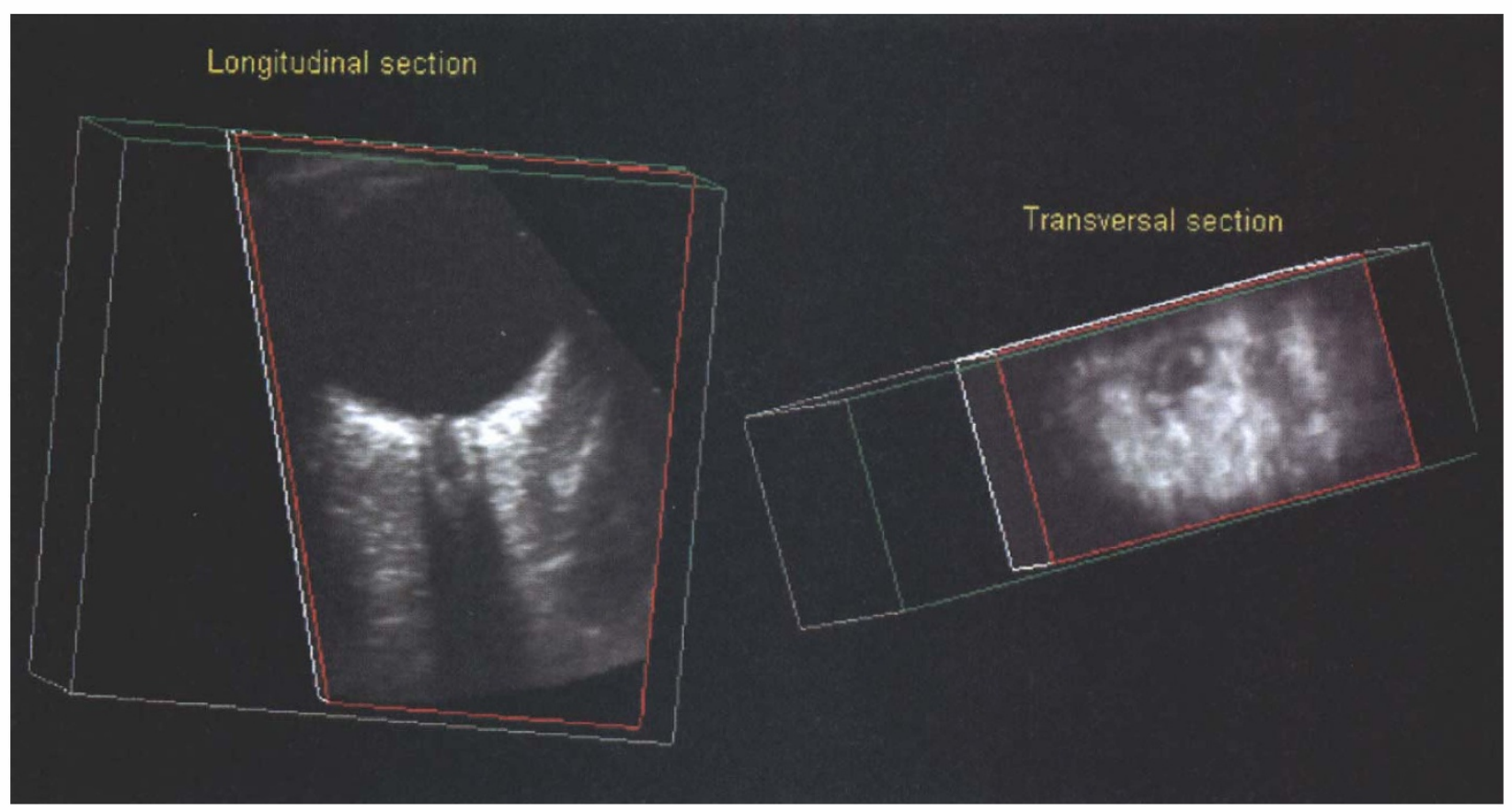

Fig. 1. A 3D reconstruction of the eye and the optic nerve. Longitudinal section with the globe, optic nerve, head of the optic nerve and longitudinal section of the lateral rectus muscle. Transverse section through the head of the optic nerve. 


\begin{tabular}{|c|c|c|c|c|c|c|}
\hline \multirow[b]{3}{*}{ Patient no. } & \multirow[b]{3}{*}{ Side } & \multicolumn{2}{|c|}{ Optic nerve } & \multirow{3}{*}{$\begin{array}{l}\text { Lateral rectus } \\
\text { muscle diameter } \\
(\mathrm{mm})\end{array}$} & \multirow{2}{*}{\multicolumn{2}{|c|}{ Vessels $^{\mathrm{a}}$}} \\
\hline & & \multirow{2}{*}{$\begin{array}{l}\text { Diameter } \\
(\mathrm{mm})\end{array}$} & \multirow{2}{*}{$\begin{array}{c}\text { Area } \\
\left(\mathrm{mm}^{2}\right)\end{array}$} & & & \\
\hline & & & & & $\mathrm{CV}$ & Cil. V. \\
\hline \multirow[t]{2}{*}{1} & $\mathrm{R}$ & 2.7 & 4.5 & 5.5 & + & + \\
\hline & $\mathrm{L}$ & 2.3 & 3.8 & 4.8 & + & + \\
\hline \multirow[t]{2}{*}{2} & $\mathbf{R}$ & 2.7 & 5.1 & 5.2 & + & + \\
\hline & $\mathrm{L}$ & 2.8 & 5.6 & 5.2 & + & + \\
\hline \multirow[t]{2}{*}{3} & $\mathrm{R}$ & 3.1 & 6.0 & 4.7 & + & + \\
\hline & $\mathrm{L}$ & 3.0 & 4.5 & 4.6 & + & + \\
\hline \multirow[t]{2}{*}{4} & $\mathrm{R}$ & 3.2 & 5.8 & 4.7 & + & + \\
\hline & $\mathrm{L}$ & 3.1 & 5.2 & 4.9 & + & + \\
\hline \multirow[t]{2}{*}{5} & $\mathrm{R}$ & 3.1 & 6.1 & 4.7 & + & + \\
\hline & $\mathrm{L}$ & 3.2 & 5.8 & 4.4 & + & + \\
\hline
\end{tabular}

$\mathrm{R}$, right; L, left.

${ }^{a} \mathrm{CV}$, central retinal vessel; Cil. V., short and long posterior ciliary vessels.

+ , intraorbital vessels visualised in colour mode.

\section{Evaluation of the intraorbital vessels}

One data acquisition was performed with power mode to evaluate the following vessels: (1) central retinal vessels, (2) short and long posterior ciliary arteries. These were classified as visualised or not visualised. Power mode was used, as software for 3D reconstruction of colourcoded data was not available.

\section{Results}

All orbits were adequately imaged by one data acquisition with the free-hand scanning of the transducer. Selected intraorbital structures were identified and their course followed, including optic nerve, central retinal arteries and veins vessels, and short

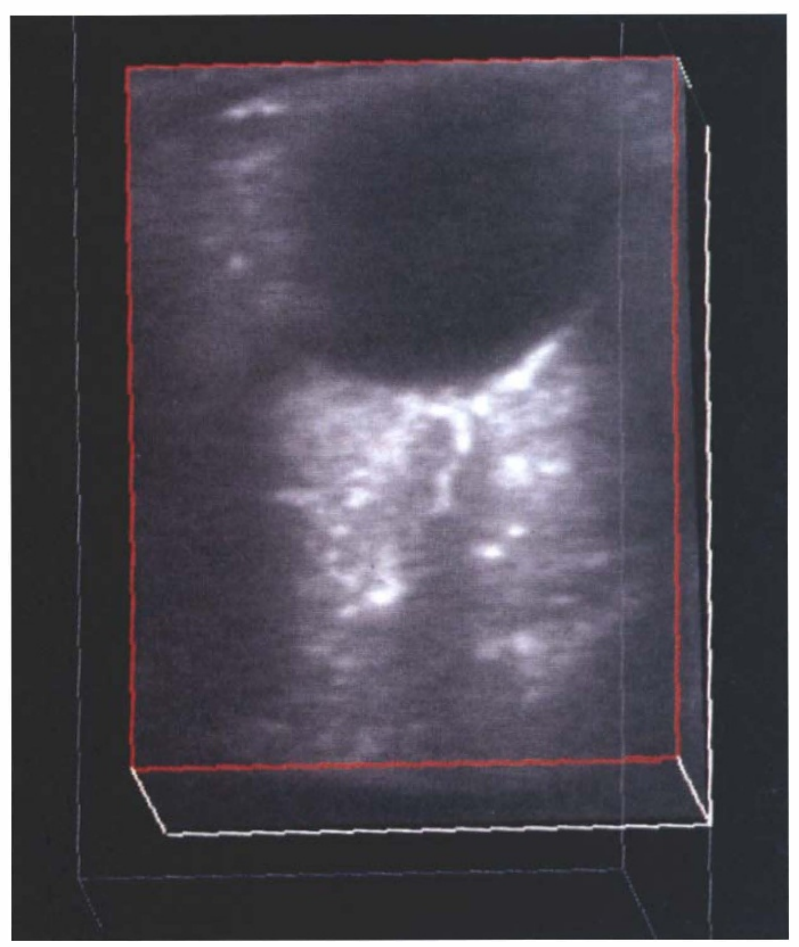

Fig. 2. Longitudinal section through the orbit and the optic nerve. In this section the central vessels of the optic nerve are clearly visible. and long posterior ciliary vessels (Table 1). The central retinal vessels were detectable in their retrolaminar portion in all 10 insonated orbits (Fig. 2). In all cases the short and the long posterior ciliary arteries could be identified.

The mean transverse area $( \pm \mathrm{SD})$ of the optic nerve was $5.6 \pm 1.1 \mathrm{~mm}^{2}$; the mean diameter $( \pm \mathrm{SD})$ of the optic nerve was $3.0 \pm 0.3 \mathrm{~mm}$ (Table 1 ). The lateral rectus muscle was clearly seen, with a mean diameter $( \pm S D)$ of $4.9 \pm 0.3 \mathrm{~mm}$.

\section{Discussion}

Standardised echography of orbit and colour duplex imaging have been limited to single views with 2D data, which may not always be adequate to assess intraorbital lesions or the relationships between different structures in the orbit. Three-dimensional ultrasound of the orbit allows some quantitative measurements (i.e. optic nerve transverse area) which are not possible with 2D images alone. In the case of diameter measurements (optic nerve, eye muscles) the location of the measurement can be more exactly determined relative to surrounding structures. Our measurements of the diameter of the optic nerve, which in the past has been measured by Ascan, nearly agree with the diameter data found by Gans et al. ${ }^{13}$ His group found a diameter with a range between 2.2 and $3.3 \mathrm{~mm}$ for a normal optic nerve.

Several studies have reported 3D ultrasound of the orbital region using a different technique (Table 2), ${ }^{5-10,12}$ but all these studies use a mechanical data acquisition system, which reduces the mobility of the transducer. Secondly, a mechanical data acquisition system needs fixation to avoid movement of the central rotation axis during data acquisition. The reduced mobility of the transducer, and the movement of the transducer out of the central rotation axis, affect the quality of the images. The magnetic sensor system for spatial data localisation avoids these problems, allowing free-hand scanning of the transducer without mechanical fixation. ${ }^{12}$ Improvements in the computer hardware and software 
Table 2. Parameters used for $3 D$ data acquisition of the eye or orbit

\begin{tabular}{|c|c|c|c|c|c|c|}
\hline Author & Year & Data acquisition ${ }^{\mathrm{a}}$ & Transducer $^{\mathrm{b}}$ & Update rate $^{c}$ & Images $^{d}$ & Time $^{e}$ \\
\hline Jensen $^{5}$ & 1991 & Mechanical rotation $180^{\circ}$ & $\begin{array}{c}10 \mathrm{MHz} \\
\text { sector }\end{array}$ & $3 / \mathrm{s}$ & 16 & $6 \mathrm{~s}$ \\
\hline Coleman $^{7}$ & 1992 & Mechanical parallel & $10 \mathrm{MHz}$ & - & - & - \\
\hline Jensen $^{6}$ & 1992 & Mechanical rotation $180^{\circ}$ & $\begin{array}{l}10 \mathrm{MHz} \\
\text { sector }\end{array}$ & $18 / \mathrm{s}$ & 20 & $2 \mathrm{~s}$ \\
\hline Silverman $^{8}$ & 1995 & Mechanical parallel & $50 \mathrm{MHz}$ & $1-2 / s$ & $16-32$ & $15-30 \mathrm{~s}$ \\
\hline Iezzi $^{10}$ & 1996 & Mechanical rotation & $50 \mathrm{MHz}$ & $8 / \mathrm{s}$ & \multicolumn{2}{|c|}{ Multiple } \\
\hline Downey $^{9}$ & 1996 & Mechanical rotation $200^{\circ}$ & $10 \mathrm{MHz}$ & $9.1 / \mathrm{s}$ & 200 & $22 \mathrm{~s}$ \\
\hline Delcker $^{12}$ & 1996 & Magnetic free-hand & $\begin{array}{l}7 / 5 \mathrm{MHz} \\
\text { sector }\end{array}$ & $11-15 / s$ & \multicolumn{2}{|c|}{$\begin{array}{l}\text { Multiple } \\
(220-450)\end{array}$} \\
\hline
\end{tabular}

${ }^{a}$ Data acquisition: Type of system used: mechanical or magnetic system with rotational or parallel movements of the transducer.

${ }^{\mathrm{b}}$ Transducer: Type and frequency of transducer used.

'Update rate: Rate of frames stored per second during data acquisition.

Images: Number of images stored for one 3D data set.

${ }^{\mathrm{e}}$ Time: Time for data acquisition of one 3D data set. Multiple: time (maximum $40 \mathrm{~s}$ ) depends on the number of the acquired 2D ultrasound sections.

required for 3D ultrasound systems have improved the ease of use and the speed of data postprocessing, and broadened the scope of potential clinical applications. In our study, data acquisition took 20-30 s, with 1-2 min (depending on the number of the stored 2D ultrasound sections) for the $3 \mathrm{D}$ reconstructions. Altogether, only $2 \frac{1}{2} \mathrm{~min}$ were required to acquire and produce the $3 \mathrm{D}$ data set.

Three-dimensional ultrasonography provides a more complete understanding of the intraorbital tissue, the spatial relationships between optic nerve, intraorbital muscles and intraorbital vessels, and continuous imaging of the tortuous path of the ophthalmic artery. The 3D approach allows retrospective visualisation, from any angle or plane. This results in greater tolerance for individual anatomical variations, more complete visualisation and understanding of intraorbital tissue structures, and more precise localisation for measurements of lesions.

\section{References}

1. Fielding JA. Pictorial review: ocular ultrasound. Clin Radiol 1996;51:533-44.

2. Erickson SJ, Hendrix LE, Massaro BM. Color Doppler flow imaging of the normal and the abnormal orbit. Radiology 1989;173:511-6.

3. Lieb WE, Cohen SM, Merton DA. Color Doppler imaging of the eye and orbit: technique and normal vascular anatomy. Arch Ophthalmol 1991;109:527-31.
4. Williamson TH, Harris A. Color Doppler ultrasound imaging of the eye and orbit. Surv Ophthalmol 1996;40:255-67.

5. Jensen PK, Hansen MK. Ultrasonographic, three-dimensional scanning for determination of intraocular tumor volume. Acta Ophthalmol (Copenh) 1991;69:178-86.

6. Jensen PK. Ultrasonographic three-dimensional scanning for determination of intraocular tumor volume. Acta Ophthalmol (Copenh) 1992;204(Suppl):23-5.

7. Coleman DJ, Silverman RH, Rondeau MJ, Lizzi FL. New perspectives: 3-D volume rendering of ocular tumors. Acta Ophthalmol (Suppl) 1992;204(Suppl):22.

8. Silverman RH, Rondeau MJ, Lizzi FL, Coleman DJ. Threedimensional high-frequency ultrasonic parameter imaging of anterior segment pathology. Ophthalmology 1995;102:837-43.

9. Downey DB, Nicolle DA, Levin MF, Fenster A. Threedimensional ultrasound imaging of the eye. Eye 1966;10:75-81.

10. Iezzi R, Rosen RB, Tello C, Liebmann J, Walsh JB, Ritch R. Personal computer-based 3-dimensional ultrasound biomicroscopy of the anterior segment. Arch Ophthalmol 1996;114:520-4.

11. Delcker A, Diener HC. Quantification of atherosclerotic plaques in carotid arteries by three-dimensional ultrasound. Br J Radiol 1994;67:672-8.

12. Delcker A, Polz H. 3D-ultrasound of carotid artery using a sensor as a space orientation. Cerebrovasc Dis 1996;6(Suppl 3):2.

13. Gans MS, Byrne SF, Glase JS. Standardised A-scan echography in optic nerve disease. Arch Ophthalmol 1987;105:1232-6. 